\title{
THE PERFORMANCE OF A TIGHT INS/GNSS/PHOTOGRAMMETRIC INTEGRATION SCHEME FOR LAND BASED MMS APPLICATIONS IN GNSS DENIED ENVIRONMENTS
}

\author{
Chien-Hsun $\mathrm{Chu}^{\text {a, } * \text {, Kai-Wei Chiang }}{ }^{\text {a }}$ \\ ${ }^{a}$ Dept. of Geomatics, National Cheng Kung University, Taiwan - chienhsun0229@msn.com
}

Commission IAG and TC I: New Trends in Direct Geo-referencing Technologies

KEY WORDS: Direct georeferencing, LC, TC, MMS

\begin{abstract}
:
Nowadays the most common technologies used for positioning and orientation of a mobile mapping system include using Global Navigation Satellite System (GNSS) as a major positioning sensor and Inertial Navigation System (INS) as the major orientation sensor. The integration strategy of the most commercially system is the loosely coupled (LC) architecture, that has the simplest architecture using the GNSS solutions to aid the INS navigation information with proper optimization estimator. The LC does combine the two sensors' solutions when the number of tracked satellite is more than four. In recent year, another commonly integration strategy is known as tightly coupled (TC) architecture. Because the TC uses the GNSS measurements to aid INS, it can integrate measurements provided by GNSS receiver and INS unless no GNSS satellite is tracked. Obviously, the TC architecture is a better candidate for land based mobile mapping applications than LC in Taiwan. Unfortunately, there are still many GNSS denied environment in the urban area, therefore the TC architecture is still not robust and stable enough for MMS application. The overall objective of this paper is to provide a scheme that tightly integrates INS/GNSS and Photogrammetric for land based MMS applications with sufficient and stable POS solutions during GNSS outages. In the traditional photogrammetry operation, numerous ground control points are applied to compute those Exterior Orientation Parameters (EOPs) of cameras by bundle adjustment. The key opinion is to derive the INS centre position and attitude and reconstruct 3-D tracking and 3-D object space by cameras EOPs. The proposed algorithm is verified using field test data collected in GNSS denied environments and the preliminary results presented in this study illustrated that the proposed algorithm is able to provide $60 \%$ improvement in terms of positioning and orientation accuracy in Taipei and Tainan cities.
\end{abstract}

\section{INTRODUCTION}

The development of land-based mobile mapping systems was initiated by two research groups, namely The Center for Mapping at Ohio State University, USA, and the Department of Geomatics Engineering at the University of Calgary, Canada. In the early 2000s, a number of land-based mobile mapping systems have been utilized in commercial applications (Chiang et al., 2008). The process of mobile mapping involves producing multiple images of a given object from various positions. The three-dimension (3D) positions of the object with respect to the camera frame can then be measured. An Inertial Navigation System (INS) is a self-contained navigation technique in which measurements provided by accelerometers and gyroscopes are used to track the position and orientation of an object relative to a known starting point, orientation and velocity. The Global Navigation Satellite Systems (GNSS) is a universal, all-weather, world-wide positioning system that provides time, position, and velocity data. Both systems can be used as stand-alone navigation tools or in conjunction with other sensors for various purposes. Moreover, the integration of GNSS and INS can overcome problems with environments such as urban canyons, forests, and indoor settings, where GNSS alone cannot provide service. In order to attain reasonable accuracies of position and orientation solutions, a tactical grade or higher quality INS along with GNSS has been applied as the primary position and orientation system for current commercial systems. Unfortunately, the cost of such systems is still at such a high level thus the popularity of mobile mapping systems remains limited, especially due to the price of the Inertial Measurement Unit (IMU). However, advances in MEMS technology enables the development of complete IMU composed of multiple MEMS-based accelerometers and gyroscopes. In addition to their compact and portable size, the price of MEMS-based systems is far less than those of high quality IMU.

In the classical approach, the KF is applied in real-time applications to fuse different data from various sensors while optimal smoothing is applied in the post-mission mode. The basic idea of using the KF in INS/GNSS integration is to fuse independent and redundant sources of navigation information with a reference navigation solution to obtain an optimal estimate of navigation states, such as position, velocity and orientation. Furthermore, for INS/GNSS integration applications where the process and measurement models are nonlinear, the Extended KF (EKF) also work for nonlinear dynamic systems with a non-Gaussian distribution, except for heavily skewed nonlinear dynamic systems, where EKF may experience problems. The EKF simply applies the first order term of the Taylor series expansion for the approximation of a nonlinear system and the probability density function is

\footnotetext{
* Corresponding author: C.H. Chu
} 
approximated by a Gaussian distribution. Only small errors are allowed during estimation and the presence of nonlinear error behavior might violate the assumption thus generates biased solutions. Second order filters are able to compensate the bias term mentioned above but the computation burden of hessian (second order derivatives) is high.

Figure 1 illustrates typical positional error accumulation of KF during GNSS outages. In fact, the error behavior of orientation parameters during GNSS outages is similar to positional error shown in Figure1.The scale of the maximum positional drift shown in Figure1 is given based on the average value of the MEMS IMU (Gyro bias $100 \mathrm{deg} / \mathrm{hr}$ ). Unfortunately, GNSS signal blockage takes place frequently in modern urban canyon. The magnitudes of the positional and orientation errors depend on the quality of the inertial sensors, the length of GNSS outage, the dynamics of vehicle and the effectiveness of the algorithms applied. In other words, proper modification of inertial sensors or sensor fusion algorithms can reduce the magnitude of accumulated positional and orientation error during frequent GNSS outages (Chiang, et al., 2011). Therefore, the goal of developing an alternative INS/GNSS integration scheme is to reduce the impact of remaining limiting factors of $\mathrm{KF}$ and improve the positioning accuracy during GNSS outages which is critical for land based mobile mapping applications.

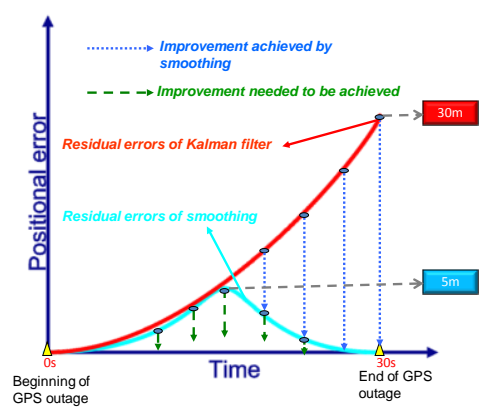

Figure 1 The KF's positional error behavior during GNSS signal blockage

Optimal smoothing algorithms, also known as smoothers, have been applied for the purpose of accurate positioning and orientation parameter determination through post-processing for most of surveying and mobile mapping applications with integrated sensors. In contrast to the $\mathrm{KF}$, the smoothing is implemented after all KF estimates have been solved by the use of past, present and future data. As shown in Figure1, the magnitudes of positional and orientation errors during GNSS outage can be improved significantly after applying one of these optimal smoothing algorithms. However, the magnitude of residual error shown with blue line also depends on the quality of the inertial sensors, the dynamics of vehicle and the length of GNSS signal outage. Therefore, the reduction of remaining positional and orientation errors becomes critical when integrating a low cost MEMS IMU with GNSS for land based mobile mapping applications.

\section{PROBLEM STATMENTS}

In order to achieve high accuracy for positioning and orientation determination in mobile mapping applications, the measurements are processed in post-mission mode with an optimal smoothing algorithm. Most of the commercial mobile mapping systems use an optimal smoothing algorithm to provide accurate information on position and orientation for direct geo-referencing (El-Sheimy, 1996). However, commercial INS/GNSS integrated systems use tactical grade IMUs or above to provide accurate solutions for general mobile mapping applications. Therefore, upgrading the hardware (e.g., IMU) can be considered as an effective solution to improve the accuracies of position and orientation parameters when a low cost MEMS IMU is used. Still, this improvement is rather limited, as the availability of high grade (navigation) IMU is regulated by the government export regulations of certain countries where those units are produced.

Another effective way to improve the accuracies of low cost MEMS INS/GNSS integrated solutions is through the improvement of sensor fusion algorithms. Compared to the hardware perspective mentioned above, the software perspective can be considered as a cost effective solution to develop a low cost sensor fusion solution for certain mobile mapping applications. Figure 2 illustrates the loosely coupled INS/GNSS integration scheme commonly applied by most of the commercial mobile mapping systems (Titterton, and Weston, 1997; Brown and Hwang, 1992). The process of the KF is divided into two groups, those for prediction and updating. The time prediction equations are responsible for the forward time transition of the current epoch (k-1) states to the next epoch $(\mathrm{k})$ states. The measurement update equations utilize new measurements into the prior state estimation to obtain an optimized posteriori state estimation. The update engine of KF is triggered at every GNSS measurement using the difference between GNSS and INS solutions as input. Hence, the KF generates an updated estimate for reducing the INS errors using measurement update equations. Whenever GNSS measurements are not available, the KF works in the time prediction mode to estimate the error state vector.The optimal smoothing is performed after the filtering stage and thus it relies on the previously filtered solutions. Consequently, an accurate filtering procedure is required for accurate smoothing process (Brown and Hwang, 1992; Gelb, 1974). A fixed-interval smoother, the Rauch-Tung-Striebel backward smoother is implemented in this study. In fixed-interval smoothing, the initial and final time epochs of the whole period of measurements (i.e. 0 and N) are fixed. Compared to other fixed-interval smoothers, the RauchTung-Striebel backward smoother has the advantage of being the simplest to implement (Brown and Hwang, 1992; Gelb, 1974). It consists of a forward sweep and a backward sweep. The forward sweep is the common KF with all predicted and updated estimates and corresponding covariance saved at each epoch of the whole mission. The backward sweep begins at the end of the forward filter (i.e. at epoch $\mathrm{N}$ ), see (Brown and Hwang, 1992; Gelb, 1974) for details.

The smoothed estimates at any epoch $\mathrm{k}$ are computed as a linear combination of the filtered estimate at that epoch and the smoothed estimate at the heading epoch $\mathrm{k}+1$. Thus, these smoothed estimates can be considered as updating the forward filtered solution to obtain improved estimates. The computation of the smoothed estimates at each epoch requires the storage of the KF predicted and updated (filtered) estimates and their corresponding covariance at each epoch (Brown and Hwang, 1992; Gelb, 1974). This is the case in INS/GNSS integrated solutions when uninterrupted GNSS data streams are available. During GNSS outages, only predicted estimates and covariance are available, a post-mission smoother can significantly remove the residual errors of KF (Titterton, and Weston, 1997; Brown and Hwang, 1992); however, some residual errors still remain, as shown in Figure2.Therefore, the error behavior shown in Figure 2 motivate various studies concerning the development 
of alternative multi-sensor fusion algorithms to reduce the magnitude of accumulated positional and orientation errors during frequent GNSS outages in land applications. This kind of integration has the benefit of a simpler architecture which is easy to utilize in navigation systems. However, the errors in the position and velocity information provided by the GNSS KF are time-correlated, which can cause degradation in performance or even instability of the navigation KF, if these correlations are not considered by some means. In the case of incomplete constellations, i.e. less than four satellites in view, the output of the GNSS receiver has to be ignored completely, leaving the INS unaided. Therefore, the current loosely coupled INS/GNSS integration architecture is not suitable for land mobile mapping applications where frequent and long GNSS outages are anticipated to deteriorate the performance of DG significantly.

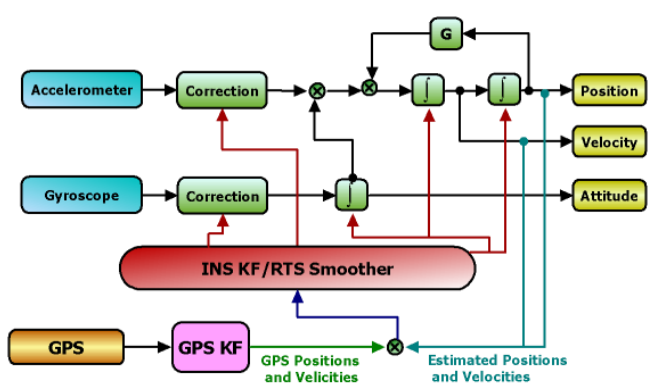

Figure 2 The integration architecture of LC (closed loop)

The tightly coupled INS/GNSS integration architecture uses a single KF to integrate GNSS and IMU measurements. Figure 3 describes the TC integration architecture. It shows that raw measurements are collected from the IMU and are converted to position, velocity and attitude measurements in the desired coordinate system using the INS mechanization algorithms. In the TC integration architecture, the GNSS pseudo-range, deltarange and carrier phase measurements are processed directly in the main Kalman filter (Hide and Moore, 2005). The aiding of the receiver tracking loops using velocity information provided by the INS is an essential characteristic of a tightly coupled system, too. The primary advantage of this integration is that raw GNSS measurements can still be used to update the INS when less than four satellites are available. This is of special benefit in a hostile environment such as downtown areas where the reception of the satellite signals is difficult due to obstruction. In addition, in the case when carrier phase GNSS measurements are used, the IMU measurements will be used to aid the ambiguity resolution algorithm. However, the TC integration architecture is not commonly used to integrate GNSS and INS simply because of its additional complexity over the LC approach. As shown in Figure 4, the positioning accuracies of TC and LC architectures remain similar when the number of visible satellite is larger than four. However, the accuracy of LC architecture decrease significantly when the number of visible satellite become less than four. On the contrary, the accuracy of TC architecture remains stable when the number of visible satellite becomes less than four. However, the accuracy of TC architecture starts to decrease when the number of visible satellite becomes less than two. Although TC architecture seems to be a better candidate for land based mobile mapping applications, but TC architecture still suffer the impact of frequent GNSS signal blockage in urban area. Figure 5 illustrates the comparison of those trajectories provided by TC architecture (shown in white), LC architecture (shown in red) and GNSS (shown in blue), respectively when the test vehicle drove under the elevated highway with limited GNSS signal visibility. Generally speaking, the trajectory of TC architecture (shown in white) remains at the right side of the road while the trajectory of LC architecture (shown in red) drifted to the opposite side of the road. Therefore, the TC architecture illustrates better robustness and stability to the immunity of GNSS signal visibility. However, since the number of visible satellite decreased to less than one for $80 \%$ of time span when the test vehicle drove under the elevated highway, the trajectory of TC architecture (shown in white) drifted to the opposite side eventually. Therefore, GNSS signal visibility and quality still play a vital role when applying TC architecture for land based mobile mapping applications. Of course none of those issues would matter when expensive and controlled navigation grade IMUs are available to general users, however, those issues are the key to improve the quality of POS solutions with the TC architecture for land mobile mapping applications using current commercially available tactical grade IMU.

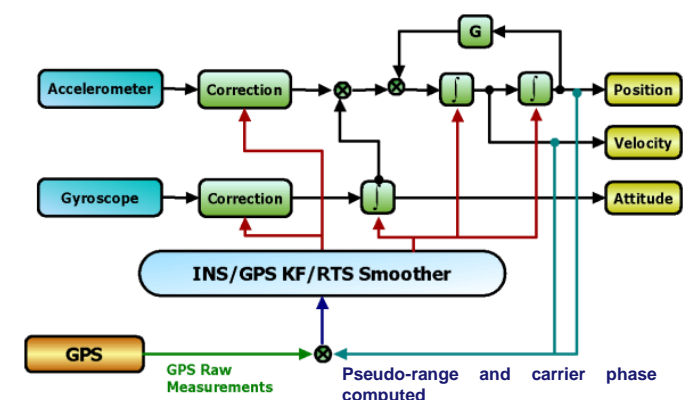

Figure 3 The integration architecture of TC (closed loop)
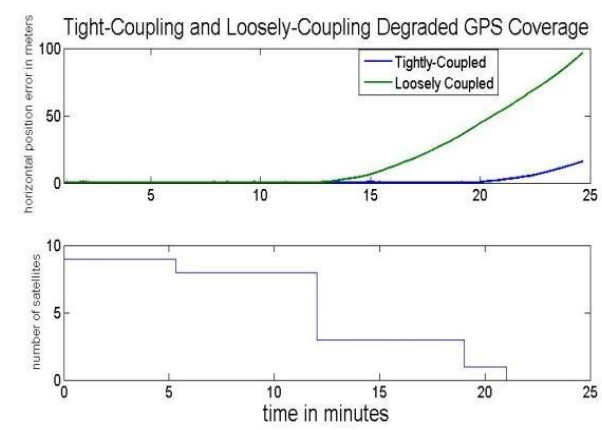

Figure 4 The comparison between TC and LC architectures with variable number of visible satellite

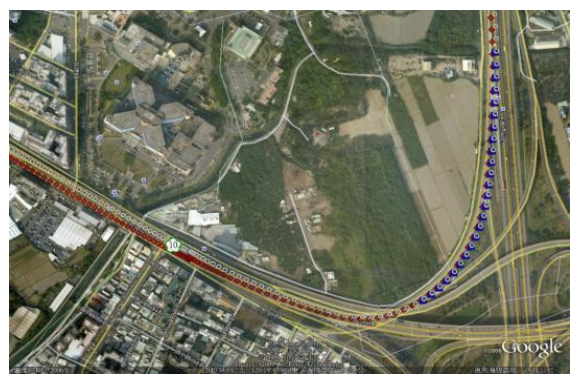

Figure 5 The comparison between TC and LC architectures in urban area

Therefore, the objective of this study is to integrate the photogrammetry technology and INS/GNSS that can overcome the limitations of current TC architecture to improve the DG accuracy in GNSS denied environments with the latest mapping van developed at the National Cheng Kung University (NCKU) in Taiwan. The novel integration scheme poses the advantages of current $\mathrm{LC}$ and TC architectures and is capable of providing sufficient robustness and stability to limited GNSS visibility 
condition for land mobile mapping applications. In addition, the performance evaluation with field test data is applied to verify the applicability of proposed algorithms.

\section{THE CONFIGURATIONS OF PROPOSED MMS}

Figure 6 illustrates the MMS van developed at the NCKU. It comprises the positioning and orientation sensors, the CCD cameras, and the time synchronization sensors. The implementation of this system is given below in details.

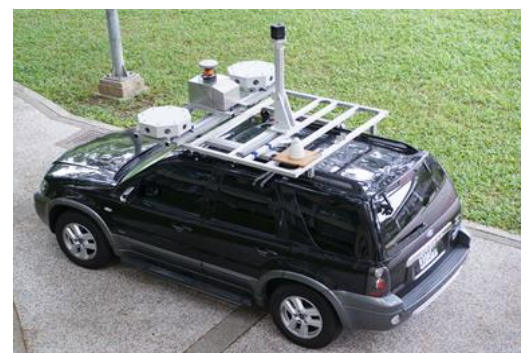

Figure 6 The proposed mobile mapping system

The original configurations of this van including the power supply and its interior designs are not suitable for mounting those sensors. Thus, several renovation works have been carried out which are illustrated as below:

In order to mount the central control PC as well as other required accessories in the vehicle, the interiors are modified as shown in Figure 7. Figure 8 also illustrates the roof rack of the proposed system. Because the vehicle can't be regarded as a rigid body, thus the roof rack is designed for mounting all those sensors required for mobile mapping applications to guarantee that all sensors works under identical environments.
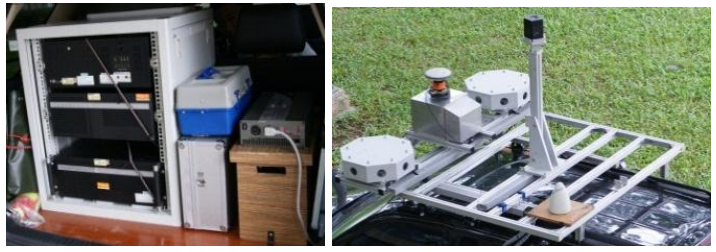

Figure 7 The interiors \& Roof Rack

The Positioning and Orientation System (POS) implemented in this study is composed of a tactical grade Inertial Measurement Unit (IMU) SPAN-CPT with a NovAtel ProPak-V3 receiver, as shown in Figure 8. The GPS measurements were collected by a duel-frequency receiver, OEM-V, which is embedded in the SPAN-CPT and a ProPak V3 receiver located on the CivilNET ${ }^{\mathrm{TM}}$ station setup by the Century Instrument Company as a base station. The raw GPS measurements are processed differentially in carrier-phase DGPS to achieve ten centimeter level accuracy using GrafNavTM 8.10 software for further processing.

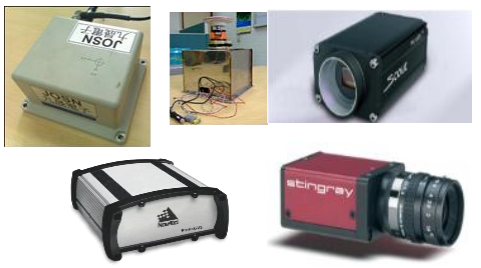

Figure 8 All sensors include SPAN-CPT system, NovAtel ProPak -V3, Basler Scout, and AVT Stingray camera.
Two Basler Scouts and two AVT Stingrary digital CCD cameras are used in this study, as shown in Figure 8. The specifications of the cameras are shown in Table 1 . The ability of image acquisition of the proposed MMS van is enhanced significantly because those cameras are equipped with electronic shutters.

\begin{tabular}{|c|c|}
\hline Cell size & $4.4 \mu \mathrm{m} * 4.4 \mu \mathrm{m}$ \\
\hline Frame rates & up to $14 \mathrm{fps}$ \\
\hline Image size & $1624 * 1234$ \\
\hline Shutter speed & $47 \mathrm{us} \sim 67 \mathrm{~s}$ \\
\hline Digital interface & IEEE1394b \\
\hline
\end{tabular}

\section{SYSTEM CALIBRATION}

This study applies a two-step approach to conduct the lever arm and the boresight calibration. The image acquisition is performed at the ground calibration field, and the measurements of the image points are also processed at this stage. Second, the Australis software is used to calculate the EOPs of those images through the bundle adjustment. After performing the interpolation of INS/GNSS data at the image exposure time, the differences of the position and the attitude between the exterior orientation parameters and the interpolated INS/GNSS data are applied. In the aspect of the lever arm calibration, the perspective position of each image $\left(\mathrm{r}_{\mathbf{C}}^{\mathrm{M}}\right)$ is exactly known after the bundle adjustment, and the calculation about the INS/GNSS position vector ( $\mathrm{r}_{\mathrm{INS} / \mathrm{GNSS}}^{\mathrm{MI}}$ ) is conducted by the interpolation at the same time. Then the lever arms ( $\mathrm{r}_{\text {INS/GNSS }}^{\mathrm{b}}$ ) can be solved by the following equation ( $\mathrm{Li}, 2010)$ :

$\mathrm{r}_{\text {INS/GNSS_a }}^{\mathrm{b}}=\mathrm{R}_{\mathrm{M}}^{\mathrm{b}}\left(\mathrm{r}_{\mathrm{c}}^{\mathrm{M}}-\mathrm{r}_{\text {INS/GNSS }}^{\mathrm{MM}}\right)$

In the aspect of the boresight calibration, the rotation matrix between the camera frame and the mapping frame of each image $\left(\mathrm{R}_{\mathrm{c}}^{\mathrm{M}}\right)$ is also obtained from the bundle adjustment results, and the rotation matrix between the body frame and mapping frame of each image can be measured by INS. The relationship is shown in Figure 9.

Eventually, the rotation matrix $\left(\mathbb{R}_{\mathrm{c}}^{\mathrm{b}}\right)$ can be calculated by the matrix multiplication ( $\mathrm{Li}, 2010)$ :

$$
\mathrm{R}_{\mathrm{c}}^{\mathrm{b}}=\mathrm{R}_{\mathrm{MI}}^{\mathrm{b}}\left(\mathrm{R}_{\mathrm{M}}^{\mathrm{c}}\right)^{-1}
$$

From those equations, the accuracy of the calibration is dominated by the quality of the INS/GNSS POS data and the bundle adjustment results. This relationship also affects DG of the MMS indirectly. In this case, the distribution of the control points in the image and the quality of the INS/GNSS data are very important in the calibration.

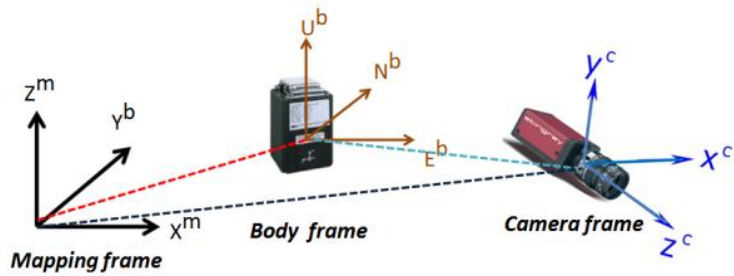

Figure 9 The relationship between INS and camera 
The calibration result of the proposed MMS Van is shown in Table 2. The lever arm relationship is around $2 \mathrm{~cm}$ and the boresight angle's accuracy is about 1 degree. In other words, that causes about $30 \mathrm{~cm}$ error for $20 \mathrm{~m}$ objects.

\begin{tabular}{|c|c|c|c|c|c|c|}
\hline & \multicolumn{3}{|c|}{ lever arm $(\mathrm{m})$} & \multicolumn{3}{c|}{ boresight(deg) } \\
\cline { 2 - 7 } & $\mathrm{x}$ & $\mathrm{y}$ & $\mathrm{z}$ & omega & phi & kappa \\
\hline AVT-A0 & 0.033 & 0.033 & 0.032 & 1.025026 & 0.274845 & 0.638329 \\
\hline AVT-A1 & 0.027 & 0.029 & 0.030 & 1.017887 & 0.276045 & 0.647716 \\
\hline Bas-A2 & 0.031 & 0.028 & 0.031 & 1.094678 & 0.276974 & 0.918387 \\
\hline Bas-A3 & 0.029 & 0.029 & 0.034 & 1.299230 & 0.254049 & 0.913461 \\
\hline Bas-A3 & 0.029 & 0.027 & 0.029 & 1.294230 & 0.272754 & 0.900304 \\
\hline Bas-A5 & 0.029 & 0.027 & 0.029 & 1.086595 & 0.269756 & 0.900439 \\
\hline
\end{tabular}

Table 2 The calibration accuracy of every camera

After obtaining calibration parameters, the DG task can be performed exactly works without GCPs. The calibration experiments and tests about DG are presented in the following sections

\section{THE INTEGRATION OF PHOTOGRAMMETRY/INS/GNSS}

Although the INS/GNSS integration system is able to perform seamlessly during GNSS outage, the accuracy degrades with GNSS outage time. In addition, frequent and long GNSS outages taking place in typical urban canyon degrade the accuracy of POS applied by the land based MMS thus deteriorate the accuracy of DG operation significantly.

In order to restrain the weaken, this study provides a method that take advantage of control points updating POS solution through photogrammetry technology. The method is also called (Multiple Tightly Coupled, MTC). The commercial software, Australis, is used to finish bundle adjustment through the control points. The Figure 10 illustrates the concept of MTC process that is the computed EOP by bundle adjustment can be integrated into INS/GNSS integration scheme for feedback during GNSS outages.

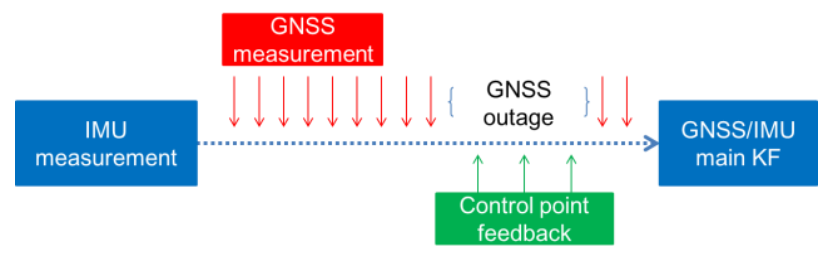

Figure 10 The concept of MTC process

The Figure 11 and 12 illustrate EOPs are derived to the IMU position and attitude for the updating in the KF. The EOPs of each image can be solved by bundle adjustment through the tiepoints and control points and then are derived into IMU position and attitude by lever-arm and boresight. Because the control points can be measured by other surveying methods in GNSS outage area and the derived position and attitude are updated in KF, the accuracy can be significantly ascended during GNSS denied environment. The EOP attitude of image and boresight angle are converted to rotation matrix by DCM for multiplication that gets rotation matrix between body frame and mapping frame. In other words, it is the rotation matrix between IMU frame and mapping frame, because the IMU frame is supposed to the completely overlapping with mapping frame. The lever-arm frame can be converted to mapping frame by the rotation matrix, and the IMU position can be calculated by the EOP position vector of image adding the converted lever-arm vector in the same coordinate system. The accuracy of POS solution can be ascend through derived position and attitude of IMU by MTC during GNSS denied environment.

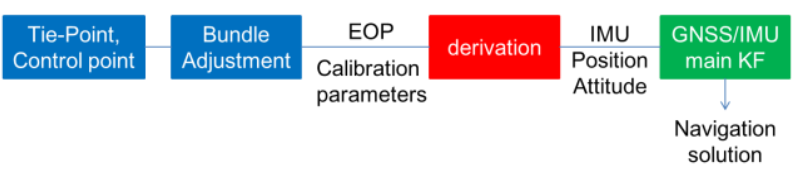

Figure 11 MTC process

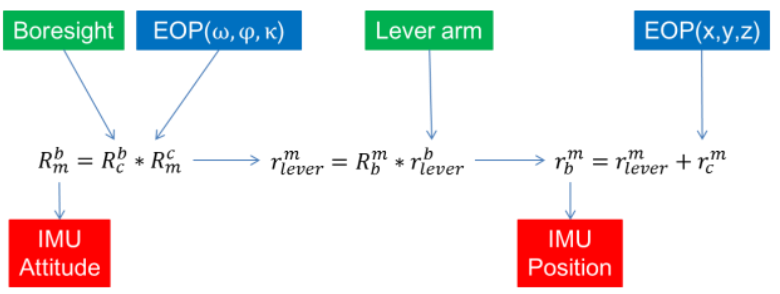

Figure 12 The detail of derivation

\section{RESULTS AND DISCUSSIONS}

This study aims at the performance between MTC and TC, because the TC is more robust and comfortable than LC in the urban. Although the TC can provide seamless POS solution, its accuracy quickly descends with time in GNSS outage. This study takes advantage of control point's feedback including position and attitude of IMU providing the updating information in KF. The whole analysis is divided into static and dynamic testing.

In the static testing, using the salved EOPs of 10 exposure stations by bundle adjustment process MTC. The Table 3 and Figure 13 illustrate the error condition of control point's feedback between test and reference tracking. In the dynamic testing, the 3 minutes GNSS outage is simulated by software for comparing TC and MTC during GNSS denied environment. The Table 4 illustrates the performance of TC and MTC; obviously, the position accuracy of MTC largely ascends about $95 \%$ in 3D. The heading accuracy ascends about $20 \%$ and the roll and pitch accuracy only ascends about $1 \%$. The Figure 14 and 15 that illustrate the more robust performance of MTC during GNSS outage are the error distribution of the whole mission. According to the result, MTC is more robust than TC during GNSS outages whatever static or dynamic testing.

\begin{tabular}{|c|c|c|c|c|}
\hline \multicolumn{2}{|c|}{ MTC } & $\mathrm{E}(\mathrm{m})$ & $\mathrm{N}(\mathrm{m})$ & $\mathrm{H}(\mathrm{m})$ \\
\hline \multirow{3}{*}{$\begin{array}{c}\text { Position } \\
\text { error }\end{array}$} & AVG & 0.0116 & 0.0309 & $1.2678 \mathrm{e}-004$ \\
\cline { 2 - 5 } & STD & 0.0165 & 0.0122 & 0.0302 \\
\cline { 2 - 5 } & RMS & 0.0194 & 0.0329 & 0.0287 \\
\hline
\end{tabular}

Table 3 The position error statistic based on reference in static testing

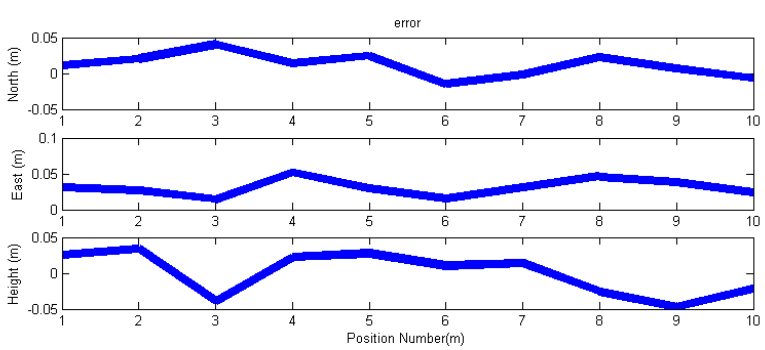

Figure 13 The position error of control point's feedback in static testing 


\begin{tabular}{|c|c|c|c|c|}
\hline \multicolumn{2}{|c|}{ TC } & \multirow{2}{*}{$\begin{array}{c}\begin{array}{c}\mathbf{E}(\mathbf{m}) / \\
\text { Roll(deg) }\end{array} \\
-0.1444 \\
\end{array}$} & \multirow{2}{*}{$\begin{array}{c}\begin{array}{c}\mathbf{N}(\mathbf{m}) / \\
\text { Pitch(deg) }\end{array} \\
-1.0086 \\
\end{array}$} & \multirow{2}{*}{ 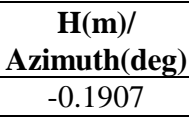 } \\
\hline Position & AVG & & & \\
\hline error & STD & 3.8355 & 10.0232 & 1.2448 \\
\hline & RMS & 3.8382 & 10.0738 & 1.2593 \\
\hline \multirow[t]{3}{*}{$\begin{array}{l}\text { Attitude } \\
\text { error }\end{array}$} & $\mathrm{AVG}$ & $\begin{array}{c}-3.8789 \mathrm{e}- \\
004\end{array}$ & $\begin{array}{c}-7.8112 \mathrm{e}- \\
005\end{array}$ & -0.0643 \\
\hline & STD & 0.0022 & 0.0022 & 0.1297 \\
\hline & RMS & 0.0022 & 0.0022 & 0.1448 \\
\hline \multicolumn{2}{|c|}{ MTC } & $\begin{array}{c}\text { E(m)/ } \\
\text { Roll(deg) }\end{array}$ & $\begin{array}{c}\mathbf{N}(\mathbf{m}) / \\
\text { Pitch(deg) }\end{array}$ & $\begin{array}{c}\mathbf{H}(\mathbf{m}) / \\
\text { Azimuth(deg) }\end{array}$ \\
\hline \multirow[t]{3}{*}{$\begin{array}{c}\text { Position } \\
\text { error }\end{array}$} & AVG & $\begin{array}{c}-3.3719 \mathrm{e}- \\
004 \\
\end{array}$ & $\begin{array}{c}.2304 \mathrm{e}- \\
005 \\
\end{array}$ & $5.9178 \mathrm{e}-004$ \\
\hline & STD & 0.0918 & 0.0937 & 0.0171 \\
\hline & RMS & 0.0918 & 0.0937 & 0.0171 \\
\hline \multirow[t]{3}{*}{$\begin{array}{l}\text { Attitude } \\
\text { error }\end{array}$} & AVG & $\begin{array}{c}-2.6895 \mathrm{e}- \\
004\end{array}$ & $\begin{array}{c}-7.0577 \mathrm{e}- \\
005\end{array}$ & -0.0428 \\
\hline & STD & 0.0020 & 0.0021 & 0.1101 \\
\hline & RMS & 0.0020 & 0.0021 & 0.1181 \\
\hline
\end{tabular}

Table 4 The error statistic of TC in dynamic test
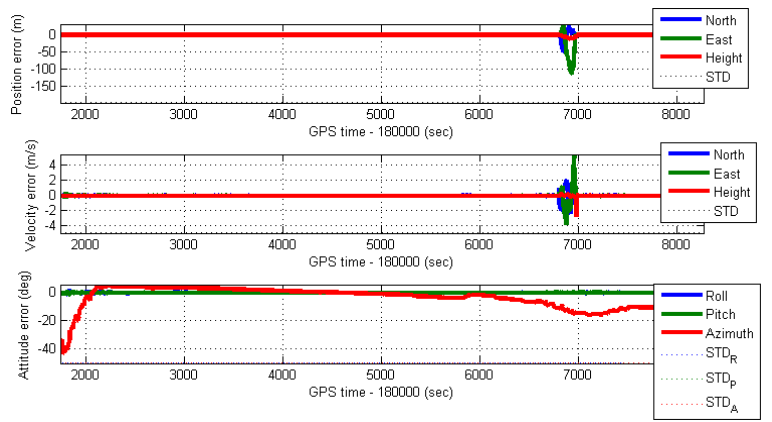

Figure 14 The TC error of POS
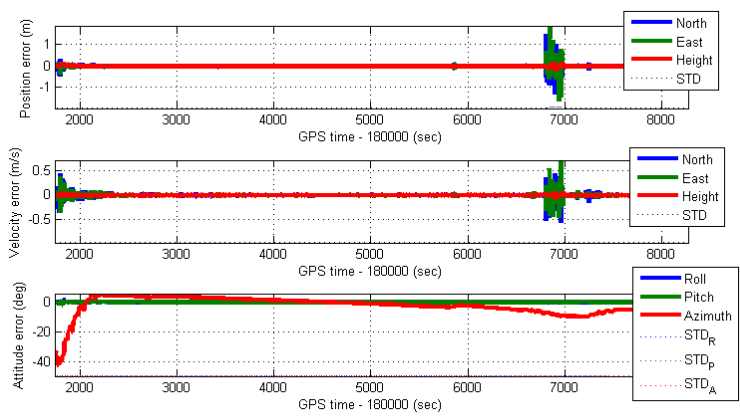

Figure 15 The MTC error of POS

\section{CONCLUSIONS}

The calibration result of the proposed MMS Van is enough to be applied on DG. The lever arm relationship is around $2 \mathrm{~cm}$ and the boresight angle's accuracy is about 1 degree. In other words, that causes about $30 \mathrm{~cm}$ error for $20 \mathrm{~m}$ objects.

Although the TC can provide seamless POS solution, its accuracy quickly descends with time in GNSS outage. This study provides MTC takes advantage of control point's feedback including position and attitude of IMU providing the updating information in KF. Moreover, this study investigated the impact of TC and proposed MTC INS/GNSS integration architectures in term of positioning accuracy using a land-based MMS van. The MTC scheme improves the 3D positioning accuracy by $95 \%$ compared to the commercially available TC architecture. The performances in terms of attitude estimation accuracy between the two architectures are similar. It proves the performance of the MTC architecture is more stable than that of the TC architectures.

\section{REFERENCES}

A. Gelb, 1974. Applied Optimal Estimation, The Analytic Science Corporation.

C. Hide, and T. Moore, 2005. GNSS and Low Cost INS Integration for Positioning in the Urban Environment, Proc. of ION GNSS 2005, pp.1007-1015

D. H. Titterton, and J. L. Weston, 1997. Strapdown Inertial Navigation Technology. Peter Peregrinus Ltd

K.W. Chiang, A. Noureldin, and N. El-Sheimy, 2008. Developing a Low Cost MEMS IMU/GNSS Integration Scheme Using Constructive Neural Networks, IEEE Transactions on Aerospace and Electronic Systems,44(2): 582-594

K.W. Chiang, H.W. Chang, C.Y. Li, and Y.W. Huang 2011. An ANN embedded RTS smoother for an INS/GPS integrated Positioning and Orientation System, Applied Soft Computing. 11(2): 2633-2644

N. El-Sheimy 1996. The development of VISAT - A mobile survey system for GIS applications. Ph.D. thesis, UCGE Report No. 20101, Department of Geomatics Engineering, the University of Calgary: Calgary, Canada.

R.G. Brown and P.Y.C. Hwang 1992. Introduction to random signals, John Wiley and Sons, New York.

Y.H. Li, 2010. The Calibration Methodology of a Low Cost Land Vehicle Mobile Mapping System, ION GNSS 2010, Portland, OR

\section{ACKNOWLEDGMENTS}

The authors would acknowledge the financial supports provided by the Department of land administration, Ministry of Interior of the Executive Yuan, R.O.C. (Taiwan).Profs. Jiann-Yeou Rau and Yi-Hsin Tseng are acknowledged to provide necessary knowledge about photogrammetry and photogrammetric related software applied in this study. 